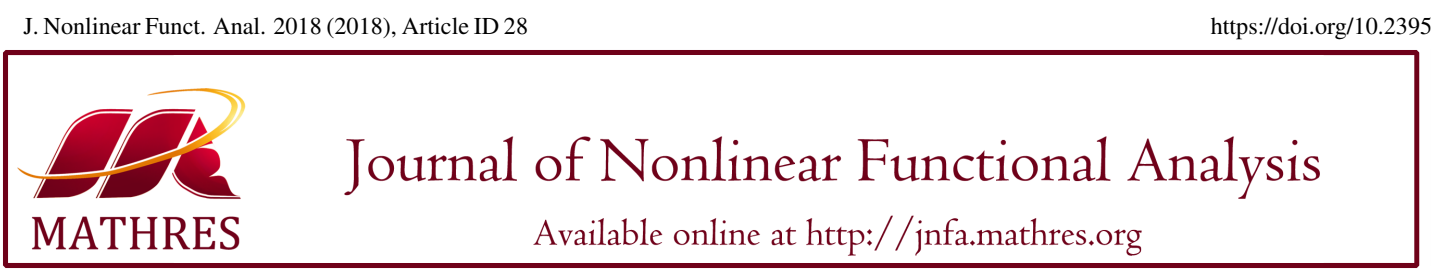

\title{
EXISTENCE AND UNIQUENESS OF SOLUTIONS FOR A CLASS OF FUNCTIONAL-INTEGRAL EQUATIONS WITH FRACTIONAL DERIVATIVE AND TIME-DEPENDENT DELAY
}

\author{
KAZEM NOURI*, MARJAN NAZARI, BAGHER KERAMATI, LEILA TORKZADEH \\ Department of Mathematics, Faculty of Mathematics, Statistics and Computer Sciences, \\ Semnan University, P. O. Box 35195-363, Semnan, Iran
}

\begin{abstract}
This paper presents the existence and uniqueness theorems of solutions for a class of fractional integro-differential equations with time-dependent delay. The Banach fixed point theorem and the Krasnoselskii's fixed point theorem are applied to establish our results. An example is provided to illustrate the effectiveness of the obtained results.

Keywords. Caputo fractional derivative; Existence and uniqueness; Fractional integro-differential equations; Krasnoselskii's fixed point theorem; Time-dependent delay.
\end{abstract}

2010 Mathematics Subject Classification. 34A12, 34K37, 26 A3.

\section{INTRODUCTION}

Fractional calculus as a branch of mathematics that deals with derivatives and integrals of arbitrary orders, appears frequently in researches due to its numerous applications in many fields of science and engineering including physics [1, 2], biology [3], chemistry [4], fluid flow, electrical network, etc [5, 6]. With the help of fractional calculus, we can formulate the mathematical models of the natural phenomena $[7,8]$. Therefore, the subject of fractional differential equations has received much attention and many papers focus on the theory [9], and their solutions [10, 11], in the last few decades. In fact, fractional derivatives have arisen as a valuable tool for the description of memory and hereditary properties of various materials and processes, the comprehensive reviews of fractional differential equations may be found in $[5,6]$.

Delay differential equations can describe many complex processes in nature and technology $[12,13]$. In fact, these equations are a special sort of functional differential equations involving past value of the state variable $[14,15]$. Functional differential equations occur often in several areas of applied

\footnotetext{
${ }^{*}$ Corresponding author.

E-mail addresses: knouri@semnan.ac.ir (K. Nouri), m.n_nazari@semnan.ac.ir (M. Nazari), bkeramati@ semnan.ac.ir (B. Keramati), torkzadeh@semnan.ac.ir (L. Torkzadeh).

Received May 25, 2018; Accepted August 23, 2018.
}

(C)2018 Journal of Nonlinear Functional Analysis 
mathematics with the delay dependent on the unknown functions [16]. These equations are nominated functional differential equations with state-dependent delay.

Recently, study on the existence of solutions for fractional differential equations with state-dependent delay becomes very attractive and many authors are studying on these problems. In [17], the existence and uniqueness solution of nonlinear fractional integro-differential equations with state-dependent delay, has been investigated on a compact interval using the measures of noncompactness technique. Hartung [18], studied well-posedness and continuous dependence issues and differentiability of solutions with respect to parameters in neutral differential equations with state-dependent delays. In [19], the existence of solutions for a class of impulsive fractional neutral stochastic integro-differential systems with nonlocal conditions and state-dependent delay, has been studied in Hilbert spaces. Suganya, Arjunan andTrujillo [20] investigated the existence of solutions for impulsive fractional integro-differential equations with state-dependent delay of the following model,

$$
\left\{\begin{array}{l}
{ }^{c} D^{\alpha} x(t)=A x(t)+f\left(t, x_{\rho\left(t, x_{t}\right)}, \int_{0}^{t} h\left(t, s, x_{\rho\left(s, x_{s}\right)}\right) d s\right), t \in[0, T]-\left\{t_{1}, t_{2}, \ldots, t_{m}\right\}, \\
\Delta x\left(t_{k}\right)=I_{k}\left(x\left(t_{k}^{-}\right)\right), k=1,2, \ldots m \\
x_{0}=\phi \in B, \quad 0<\alpha<1 .
\end{array}\right.
$$

They obtained their results with the help of different fixed point theorems, Banach contraction principle, Krasnoselskii's and Schaefer's fixed point theorems.

In our previous paper [21], we investigated the existence of solutions for the class of fractional integrodifferential equations with time-dependent delay. Using the Krasnoselskii's fixed point theorem, we obtained our results for a coupled system of fractional integro-differential equations with time-dependent delay of the following form

$$
\left\{\begin{aligned}
{ }^{c} D^{\alpha}\left[x(t)-l_{1}\left(t, x_{\rho(t)}\right)\right]= & I^{1-\alpha} f_{1}\left(t, x_{\rho(t)}, K_{1} y(t)\right) \\
& +F_{1}\left(t, y_{t}, \int_{0}^{t}(t-s)^{\alpha-1} h_{1}\left(t, s, x_{s}\right) d s\right), \\
{ }^{c} D^{\alpha}\left[y(t)-l_{2}\left(t, y_{\rho(t)}\right)\right]= & I^{1-\alpha} f_{2}\left(t, y_{\rho(t)}, K_{2} x(t)\right) \\
& +F_{2}\left(t, x_{t}, \int_{0}^{t}(t-s)^{\alpha-1} h_{2}\left(t, s, y_{s}\right) d s\right), \\
t \in J=[0, T], \quad x_{0}=y_{0}= & \phi \in B .
\end{aligned}\right.
$$

In this paper, we discuss the existence and uniqueness of solutions for a class of fractional integrodifferential equations with time-dependent delay of the following form

$$
\left\{\begin{aligned}
&{ }^{c} D^{\alpha}\left[x(t)-g\left(t, x_{\rho(t)}\right)\right]= f_{1}\left(t, x_{t}, \int_{0}^{t}(t-s)^{(\alpha-1)} x(s) d s\right) \\
& \times f_{2}\left(t, x_{\rho(t)}, \int_{0}^{t} h\left(s, x_{s}\right) d s\right), \\
& x_{0}=\phi \in B, \quad t \in J=[0, T], \quad 0<\alpha \leq 1,
\end{aligned}\right.
$$

where ${ }^{c} D^{\alpha}$ is the Caputo fractional derivative, $f_{1}, f_{2}: J \times B \times \mathbb{X} \rightarrow \mathbb{X}, g, h: J \times B \rightarrow \mathbb{X}$, and $\rho: J \rightarrow$ $(-\infty, T]$ are given functions satisfying some appropriate hypotheses. Also, $B$ is a phase space of mapping $(-\infty, 0]$ into $\mathbb{X}$ which will be defined in the next section. For each $x:(-\infty, T] \rightarrow \mathbb{X}$, the function $x_{t}$ : $(-\infty, 0] \rightarrow \mathbb{X}$ is defined by $x_{t}(\theta)=x(t+\theta)$ for $\theta \in(-\infty, 0], t \in[0, T]$. 
In contrast to the current outcomes, some positive aspects will be presented in this paper: First of all, we investigate the existence and uniqueness results of solutions for a class of fractional integrodifferential equations with time-dependent delays that include the product of two nonlinear operators $f_{1}, f_{2}$. Because of the inappropriateness of using function $\rho\left(t, x_{t}\right)$ in the previous related works, we replace $\rho\left(t, x_{t}\right)$ with $\rho(t)$ to obtain our results, and we present a new definition of function $\rho\left(t, x_{t}\right)$ for the proof of the contraction mapping in our paper. Then, we implement the Banach and Krasnoselskii's fixed point theorems to examine model (1.1).

The structure of the paper is as follows. In Section 2, we present some preliminaries and lemmas that be used to prove our main results. In Section 3, the existence of solutions for problem (1.1) is analyzed under the Banach fixed point theorem and the Krasnoselskii's fixed point theorem. In Section 4, an example is given to illustrate our results. Finally, the paper is ended with a brief conclusion in Section 5.

\section{PRELIMINARIES}

In this section, we recall some preliminary components and notations which are used throughout of this paper. These concepts can be found in [6, 22, 23].

Let $\mathbb{X}$ be a Banach space with the standard norm $\|$.$\| , and let C(J, \mathbb{X})$ denote the Banach space of all continuous functions from $J$ into $\mathbb{X}$ with the norm

$$
\|f\|_{C(J, \mathbb{X})}=\sup _{0 \leq t \leq T}\|f(t)\| .
$$

Let $L^{1}(J, \mathbb{X})$ be the Banach space of measurable functions from $J$ into $\mathbb{X}$ which are Lebesgue integrable with the norm

$$
\|f\|_{L^{1}}=\int_{J}\|f(t)\| d t .
$$

Definition 2.1. ([6]) The Riemann-Liouville fractional integral of order $\alpha>0$ of the function $f \in$ $L^{1}(J, \mathbb{X})$ is defined as

$$
I^{\alpha} f(t)=\frac{1}{\Gamma(\alpha)} \int_{0}^{t}(t-\tau)^{\alpha-1} f(\tau) d \tau
$$

where $\Gamma($.$) is the Gamma function.$

Definition 2.2. ([6]) The $\alpha$-th Caputo fractional derivative of $f \in C(J, \mathbb{X})$ is defined as

$$
{ }^{c} D^{\alpha} f(t)=\frac{1}{\Gamma(n-\alpha)} \int_{0}^{t}(t-\tau)^{n-\alpha-1} f^{(n)}(\tau) d \tau, n-1<\alpha<n, n \in \mathbb{N} .
$$

Lemma 2.3. (Krasnoselskii's fixed point theorem). Let $\mathbb{X}$ be a Banach space and let $E$ be a bounded, closed, convex subset of $\mathbb{X}$. Let $S_{1}, S_{2}$ be maps of $E$ into $\mathbb{X}$ such that $\left(S_{1}+S_{2}\right) x \in E$ for each $x \in E$. If $S_{1}$ is a contraction mapping and $S_{2}$ is completely continuous, then the equation

$$
S_{1} x+S_{2} x=x
$$

has at least one solution on $E$.

By means of the Krasnoselskii's fixed point theorem, we will present some results for problem (1.1) in the next section.

To describe the fractional integro-differential equations with time-dependent delay, we introduce the phase space $\left(B,\|\cdot\|_{B}\right)$, which is the vector space of functions defined from $(-\infty, 0]$ into $\mathbb{X}$ with a seminorm 
$\|\cdot\|_{B}$ such that the following axioms hold [23]:

(A) For every $f:(-\infty, T] \rightarrow \mathbb{X}$ with $f_{0} \in B$ and $t \in J$ :

$$
\begin{aligned}
& \text { (I) } f_{t} \in B, \\
& \text { (II) }\left\|f_{t}\right\|_{B} \leq N(t) \sup _{0 \leq s \leq t}\|f(s)\|+M(t)\left\|f_{0}\right\|_{B}, \\
& \text { (III) }\|f(t)\| \leq h\left\|f_{t}\right\|_{B},
\end{aligned}
$$

where $h>0$ is a constant, $N \in C(J,(0, \infty)), M: J \rightarrow(0, \infty)$ is locally bounded, and $h, N, M$ are independent of $f($.$) . Also, we assume$

$$
n_{T}=\sup _{0 \leq t \leq T}|N(t)|, \text { and } m_{T}=\sup _{0 \leq t \leq T}|M(t)|
$$

(A-1) For the function $f($.$) satisfies the conditions (A), the function t \rightarrow f_{t}$ from $J$ into $B$ is continuous. (B) The space $B$ is complete.

Remark 2.4. Condition (A)-(III) is equivalent to $\|\phi(0)\| \leq h\|\phi\|_{B}$.

Remark 2.5. ([22]) Let $f_{0} \in B$ and $t<0$. The notation $\left(f_{0}\right)_{t}$ represents the function defined by $\left(f_{0}\right)_{t}(\theta)=$ $f_{0}(\theta+t), \theta \in(-\infty, 0]$. Therefore, for the function $f($.$) in axiom (A)$, we get $f_{t}=\left(f_{0}\right)_{t}, t<0$. Obviously, $\left(f_{0}\right)_{t}$ is well defined for $t<0$, since the domain of $f_{0}($.$) is (-\infty, 0]$.

In addition, we give the following hypothesis on $f_{t}, t<0$ :

$\left(H_{f_{0}}\right)$ Let $\mathfrak{L}\left(\rho^{-}\right)=\{\rho(t): \rho(t)<0, t \in J\}$. The function $t \rightarrow f_{t}$ is well defined and continuous from $\mathfrak{L}\left(\rho^{-}\right)$into $B$, and there exists a continuous and bounded function $J^{f_{0}}(t): \mathfrak{L}\left(\rho^{-}\right) \rightarrow(0, \infty)$ such that, for each $t \in \mathfrak{L}\left(\rho^{-}\right)$,

$$
\left\|f_{t}\right\|_{B} \leq J^{f_{0}}(t)\left\|f_{0}\right\|_{B} .
$$

Lemma 2.6. ([22]) Let $f:(-\infty, T] \rightarrow \mathbb{X}$ be the continuous function on $J$ such that $f_{0} \in B$ and condition $\left(H_{f_{0}}\right)$ holds, then

$$
\left\|f_{t}\right\|_{B} \leq\left(m_{T}+J^{f_{0}}\right)\left\|f_{0}\right\|_{B}+n_{T} \sup _{0 \leq s \leq t}\|f(s)\|, t \in \mathfrak{L}\left(\rho^{-}\right) \cup J
$$

where $J^{f_{0}}=\sup _{t \in \mathfrak{L}\left(\rho^{-}\right)} J^{f_{0}}(t)$.

We consider the space

$$
\Omega=\left\{f:(-\infty, T] \rightarrow \mathbb{X},\left.f\right|_{[0, T]} \in C(J, \mathbb{X}), f_{0}=\phi \in B\right\}
$$

equipped with norm

$$
\|f\|_{\Omega}=\left\|f_{0}\right\|_{B}+\sup _{0 \leq t \leq T}\|f(t)\|
$$

which is a seminorm in $\Omega$. 


\section{EXISTENCE AND UNIQUENESS OF THE SOLUTION}

In this section, we demonstrate the existence and uniqueness theorems of solutions to problem (1.1). To obtain the main results, we need the following assumptions:

$\left(H_{1}\right): g, h: J \times B \rightarrow \mathbb{X}$ are the continuous functions, and there exist the constants $g_{1}, h_{1}>0$ such that

$$
\begin{array}{ll}
\left\|g\left(t, x_{1}\right)-g\left(t, x_{2}\right)\right\| \leq g_{1}\left\|x_{1}-x_{2}\right\|_{B}, & g^{*}=\sup _{t \in J}\|g(t, 0)\|, \\
\left\|h\left(t, x_{1}\right)-h\left(t, x_{2}\right)\right\| \leq h_{1}\left\|x_{1}-x_{2}\right\|_{B}, & h^{*}=\sup _{t \in J}\|h(t, 0)\| .
\end{array}
$$

$\left(H_{2}\right): f_{i}: J \times B \times \mathbb{X} \rightarrow \mathbb{X}, i=1,2$, are the continuous functions, and there exist the constants $f_{i j}, i=$ $1,2, j=1,2$, such that

$$
\begin{aligned}
& \left\|f_{i}\left(t, x_{1}, y_{1}\right)-f_{i}\left(t, x_{2}, y_{2}\right)\right\| \leq f_{i 1}\left\|x_{1}-x_{2}\right\|_{B}+f_{i 2}\left\|y_{1}-y_{2}\right\|, \\
& f_{i}^{*}=\sup _{t \in J}\left\|f_{i}(t, 0,0)\right\|, \quad i=1,2 .
\end{aligned}
$$

Lemma 3.1. A function $x \in \Omega$ is a solution of problem (1.1), if it satisfies the following integral equation:

$$
\begin{aligned}
& x(t)= \phi(0)-g\left(0, x_{\rho(0)}\right)+g\left(t, x_{\rho(t)}\right) \\
&+\frac{1}{\Gamma(\alpha)} \int_{0}^{t}(t-s)^{(\alpha-1)} f_{1}\left(s, x_{s}, \int_{0}^{s}(s-\gamma)^{(\alpha-1)} x(\gamma) d \gamma\right) \\
& \quad \times f_{2}\left(s, x_{\rho(s)}, \int_{0}^{s} h\left(\gamma, x_{\gamma}\right) d \gamma\right) d s
\end{aligned}
$$

Proof. Assume that $x$ is a solution of (1.1). For every $t \in J$, we have

$$
\begin{aligned}
{ }^{c} D^{\alpha}\left[x(t)-g\left(t, x_{\rho(t)}\right)\right]= & f_{1}\left(t, x_{t}, \int_{0}^{t}(t-s)^{(\alpha-1)} x(s) d s\right) \\
& \times f_{2}\left(t, x_{\rho(t)}, \int_{0}^{t} h\left(s, x_{s}\right) d s\right) .
\end{aligned}
$$

Applying the Riemann-Liouville fractional integral operator of order $\alpha$ on both sides, we obtain

$$
\begin{aligned}
& x(t)-g\left(t, x_{\rho(t)}\right)+c_{1} \\
& =\frac{1}{\Gamma(\alpha)} \int_{0}^{t}(t-s)^{(\alpha-1)} \times\left(f_{1}\left(s, x_{s}, \int_{0}^{s}(s-\gamma)^{(\alpha-1)} x(\gamma) d \gamma\right) \cdot f_{2}\left(s, x_{\rho(s)}, \int_{0}^{s} h\left(\gamma, x_{\gamma}\right) d \gamma\right)\right) d s
\end{aligned}
$$

Using the initial condition, we get

$$
c_{1}=-\phi(0)+g\left(0, x_{\rho(0)}\right)
$$

which implies

$$
\begin{aligned}
x(t)= & \phi(0)-g\left(0, x_{\rho(0)}\right)+g\left(t, x_{\rho(t)}\right) \\
+\frac{1}{\Gamma(\alpha)} & \int_{0}^{t}(t-s)^{(\alpha-1)} f_{1}\left(s, x_{s}, \int_{0}^{s}(s-\gamma)^{(\alpha-1)} x(\gamma) d \gamma\right) \\
& \times f_{2}\left(s, x_{\rho(s)}, \int_{0}^{s} h\left(\gamma, x_{\gamma}\right) d \gamma\right) d s .
\end{aligned}
$$

Conversely, $x$ is a solution of integral equation (3.1). This completes the proof. 
Theorem 3.2. Suppose that conditions $\left(H_{1}\right)$ and $\left(H_{2}\right)$ are satisfied. With the appropriate choice for $\mu_{i}, i=1,2, \cdots, 4$, and $\lambda_{1}$

$$
\begin{aligned}
\lambda_{1}= & \left(g_{1} n_{T}+\frac{T^{\alpha}}{\Gamma(\alpha+1)}\left[\left(f_{11} \mu_{1}+f_{12} \mu_{3}+f_{1}^{*}\right)\left(f_{21} n_{T}+T f_{22} h_{1} n_{T}\right)\right]\right. \\
& \left.+\frac{T^{\alpha}}{\Gamma(\alpha+1)}\left[\left(f_{21} \mu_{2}+f_{22} \mu_{4}+f_{2}^{*}\right)\left(f_{11} n_{T}+\frac{T^{\alpha}}{\alpha} f_{12}\right)\right]\right)<1,
\end{aligned}
$$

problem (1.1) has a unique solution.

Proof. First, in order to prove this theorem, we need to transform problem (1.1) into a fixed point problem. Therefore, with the help of Lemma 3.1, we define the operator $N: \Omega \rightarrow \Omega$ as

$$
N x(t)=\left\{\begin{array}{l}
\phi(0)-g\left(0, x_{\rho(0)}\right)+g\left(t, x_{\rho(t)}\right)+\frac{1}{\Gamma(\alpha)} \int_{0}^{t}(t-s)^{(\alpha-1)} \\
\quad \times\left(f_{1}\left(s, x_{s}, \int_{0}^{s}(s-\gamma)^{(\alpha-1)} x(\gamma) d \gamma\right) \cdot f_{2}\left(s, x_{\rho(s)}, \int_{0}^{s} h\left(\gamma, x_{\gamma}\right) d \gamma\right)\right) d s, \quad t \in J \\
\phi(t), t \in(-\infty, 0] .
\end{array}\right.
$$

We express the function $\varphi():.(-\infty, T] \rightarrow \mathbb{R}$ by

$$
\varphi(t)= \begin{cases}\phi(0), & t \in J, \\ \phi(t), & t \in(-\infty, 0] .\end{cases}
$$

Let $x(t)=y(t)+\varphi(t), t \in(-\infty, T]$. It is evident that $x$ satisfies in equation (3.1) if and only if $y_{0}=0$. Also, it suggests $x_{\rho(t)}=y_{\rho(t)}+\varphi_{\rho(t)}, x_{t}=y_{t}+\varphi_{t}, t \in J$, and

$$
\begin{aligned}
y(t)=- & g\left(0, x_{\rho(0)}\right)+g\left(t, y_{\rho(t)}+\varphi_{\rho(t)}\right) \\
+\frac{1}{\Gamma(\alpha)} & \int_{0}^{t}(t-s)^{(\alpha-1)}\left(f_{1}\left(s, y_{s}+\varphi_{s}, \int_{0}^{s}(s-\gamma)^{(\alpha-1)}(y+\varphi)(\gamma) d \gamma\right)\right. \\
& \left.\times f_{2}\left(s, y_{\rho(s)}+\varphi_{\rho(s)}, \int_{0}^{s} h\left(\gamma, y_{\gamma}+\varphi_{\gamma}\right) d \gamma\right)\right) d s .
\end{aligned}
$$

Let $\hat{\Omega}=\left\{y \in \Omega: y_{0}=0 \in B\right\}$, and $\|\cdot\|_{\hat{\Omega}}$ be the seminorm in $\hat{\Omega}$ described by

$$
\|y\|_{\hat{\Omega}}=\left\|y_{0}\right\|_{B}+\sup _{0 \leq t \leq T}\|y(t)\|=\|y\|_{C(J, \mathbb{X})}
$$

as a result, $\left(\hat{\Omega},\|\cdot\|_{\hat{\Omega}}\right)$ is a Banach space. We define the operator $\bar{N}: \hat{\Omega} \rightarrow \hat{\Omega}$ as follows

$$
\begin{aligned}
\bar{N} y(t)= & -g\left(0, x_{\rho(0)}\right)+g\left(t, y_{\rho(t)}+\varphi_{\rho(t)}\right) \\
& +\frac{1}{\Gamma(\alpha)} \int_{0}^{t}(t-s)^{(\alpha-1)}\left(f_{1}\left(s, y_{s}+\varphi_{s}, \int_{0}^{s}(s-\gamma)^{(\alpha-1)}(y+\varphi)(\gamma) d \gamma\right)\right. \\
& \times\left(f_{2}\left(s, y_{\rho(s)}+\varphi_{\rho(s)}, \int_{0}^{s} h\left(\gamma, y_{\gamma}+\varphi_{\gamma}\right) d \gamma\right)\right) d s
\end{aligned}
$$

It is clear that the operator $\bar{N}$ has a fixed point, if and only if $N$ has a fixed point. Thus, the aim is to show that $\bar{N}$ has a fixed point.

Choose

$$
R \geq\left\|g\left(0, x_{\rho(0)}\right)\right\|+\mu_{5}+\frac{T^{\alpha}}{\Gamma(\alpha+1)}\left(f_{11} \mu_{1}+f_{12} \mu_{3}+f_{1}^{*}\right)\left(f_{21} \mu_{2}+f_{22} \mu_{4}+f_{2}^{*}\right),
$$


and $D_{R}=\left\{y \in \hat{\Omega}:\|y\|_{\hat{\Omega}} \leq R\right\}$. Indeed, $D_{R} \subseteq \hat{\Omega}$ is closed, bounded and convex subset in $\hat{\Omega}$. We prove that $\bar{N}\left(D_{R}\right) \subseteq D_{R}$. By means of the continuity of the nonlinear functions $f_{1}, f_{2}, h$, and $g$, it can be shown that $\bar{N}$ is continuous in $D_{R}$.

From the assumption (A)-(II) and Lemma 2.6, we get the following estimates

$$
\begin{aligned}
\left\|y_{s}+\varphi_{s}\right\|_{B} & \leq m_{T}\left\|y_{0}\right\|_{B}+n_{T} \sup _{0 \leq \eta \leq s}\|y(\eta)\|+m_{T}\|\phi\|_{B}+n_{T}\|\phi(0)\| \\
& \leq n_{T} R+m_{T}\|\phi\|_{B}+n_{T}\|\phi(0)\|=\mu_{1},
\end{aligned}
$$

and

$$
\begin{aligned}
\left\|y_{\rho(s)}+\varphi_{\rho(s)}\right\|_{B} & \leq\left\|y_{\rho(s)}\right\|_{B}+\left\|\varphi_{\rho(s)}\right\|_{B} \\
& \leq\left(m_{T}+J^{y_{0}}\right)\left\|y_{0}\right\|_{B}+n_{T} \sup _{0 \leq \eta \leq s}\|y(\eta)\|+\left(m_{T}+J^{\phi}\right)\|\phi\|_{B}+n_{T}\|\phi(0)\| \\
& \leq n_{T} R+\left(m_{T}+J^{\phi}\right)\|\phi\|_{B}+n_{T}\|\phi(0)\|=\mu_{2} .
\end{aligned}
$$

Also, we have

$$
\left\|\int_{0}^{s}(s-\gamma)^{(\alpha-1)}(y+\varphi)(\gamma) d \gamma\right\| \leq \frac{T^{\alpha}}{\alpha}(R+\|\phi(0)\|)=\mu_{3} .
$$

Applying $\left(H_{1}\right)$, we obtain

$$
\left\|\int_{0}^{s} h\left(\gamma, y_{\gamma}+\varphi_{\gamma}\right) d \gamma\right\| \leq T\left\|h\left(\gamma, y_{\gamma}+\varphi_{\gamma}\right)\right\| \leq T\left(\mu_{1} h_{1}+h^{*}\right)=\mu_{4},
$$

and

$$
\left\|g\left(t, y_{\rho(t)}+\varphi_{\rho(t)}\right)\right\| \leq g_{1} \mu_{2}+g^{*}=\mu_{5} .
$$

For every $y \in D_{R}$, by means of $\left(H_{2}\right)$ and triangle inequality, we get

$$
\begin{aligned}
\| f_{1} & \left(s, y_{s}+\varphi_{s}, \int_{0}^{s}(s-\gamma)^{(\alpha-1)}(y+\varphi)(\gamma) d \gamma\right) \| \\
\leq & \left\|f_{1}\left(s, y_{s}+\varphi_{s}, \int_{0}^{s}(s-\gamma)^{(\alpha-1)}(y+\varphi)(\gamma) d \gamma\right)-f_{1}(s, 0,0)\right\| \\
& \quad+\left\|f_{1}(s, 0,0)\right\| \\
\leq & f_{11}\left\|y_{s}+\varphi_{s}\right\|_{B}+f_{12}\left\|\int_{0}^{s}(s-\gamma)^{(\alpha-1)}(y+\varphi)(\gamma) d \gamma\right\|+f_{1}^{*} \\
\leq & f_{11} \mu_{1}+f_{12} \mu_{3}+f_{1}^{*},
\end{aligned}
$$

and

$$
\begin{aligned}
\| f_{2}( & \left.s, y_{\rho(s)}+\varphi_{\rho(s)}, \int_{0}^{s} h\left(\gamma, y_{\gamma}+\varphi_{\gamma}\right) d \gamma\right) \| \\
\leq & \left\|f_{2}\left(s, y_{\rho(s)}+\varphi_{\rho(s)}, \int_{0}^{s} h\left(\gamma, y_{\gamma}+\varphi_{\gamma}\right) d \gamma\right)-f_{2}(s, 0,0)\right\| \\
& \quad+\left\|f_{2}(s, 0,0)\right\| \\
\leq & f_{21}\left\|y_{\rho(s)}+\varphi_{\rho(s)}\right\|_{B}+f_{22}\left\|\int_{0}^{s} h\left(\gamma, y_{\gamma}+\varphi_{\gamma}\right) d \gamma\right\|+f_{2}^{*} \\
\leq & f_{21} \mu_{2}+f_{22} \mu_{4}+f_{2}^{*} .
\end{aligned}
$$


Now, we combine the above estimations to show that $\bar{N}\left(D_{R}\right) \subseteq D_{R}$

$$
\begin{aligned}
\|\bar{N} y(t)\| \leq & \left\|g\left(0, x_{\rho(0)}\right)\right\|+\left\|g\left(t, y_{\rho(t)}+\varphi_{\rho(t)}\right)\right\|+\frac{1}{\Gamma(\alpha)} \int_{0}^{t}(t-s)^{(\alpha-1)} \\
& +\frac{1}{\Gamma(\alpha)} \int_{0}^{t}(t-s)^{(\alpha-1)}\left\|f_{1}\left(s, y_{s}+\varphi_{s}, \int_{0}^{s}(s-\gamma)^{(\alpha-1)}(y+\varphi)(\gamma) d \gamma\right)\right\| \\
& \times\left\|f_{2}\left(s, y_{\rho(s)}+\varphi_{\rho(s)}, \int_{0}^{s} h\left(\gamma, y_{\gamma}+\varphi_{\gamma}\right) d \gamma\right)\right\| d s \\
\leq & \left\|g\left(0, x_{\rho(0)}\right)\right\|+\mu_{5}+\frac{T^{\alpha}}{\Gamma(\alpha+1)}\left(f_{11} \mu_{1}+f_{12} \mu_{3}+f_{1}^{*}\right)\left(f_{21} \mu_{2}+f_{22} \mu_{4}+f_{2}^{*}\right) .
\end{aligned}
$$

Thus, $\|\bar{N} y\|_{\hat{\Omega}} \leq R$.

Next, we show that $\bar{N}$ is the contraction mapping. For $y(t), z(t) \in D_{R}$ and $t \in[0, T]$, we obtain

$$
\begin{aligned}
& \|\bar{N} y(t)-\bar{N} z(t)\| \leq\left\|g\left(t, y_{\rho(t)}+\varphi_{\rho(t)}\right)-g\left(t, z_{\rho(t)}+\varphi_{\rho(t)}\right)\right\|+\frac{1}{\Gamma(\alpha)} \int_{0}^{t}(t-s)^{(\alpha-1)} \\
& \times\left(\|\left\{f_{1}\left(s, y_{s}+\varphi_{s}, \int_{0}^{s}(s-\gamma)^{(\alpha-1)}(y+\varphi)(\gamma) d \gamma\right)\right.\right. \\
& \left.\times f_{2}\left(s, y_{\rho(s)}+\varphi_{\rho(s)}, \int_{0}^{s} h\left(\gamma, y_{\gamma}+\varphi_{\gamma}\right) d \gamma\right)\right\} \\
& -\left\{f_{1}\left(s, z_{s}+\varphi_{s}, \int_{0}^{s}(s-\gamma)^{(\alpha-1)}(z+\varphi)(\gamma) d \gamma\right)\right. \\
& \left.\left.\times f_{2}\left(s, z_{\rho(s)}+\varphi_{\rho(s)}, \int_{0}^{s} h\left(\gamma, z_{\gamma}+\varphi_{\gamma}\right) d \gamma\right)\right\} \| d s\right) \\
& \leq g_{1}\left\|y_{\rho(t)}-z_{\rho(t)}\right\|_{B}+\frac{1}{\Gamma(\alpha)} \int_{0}^{t}(t-s)^{(\alpha-1)} \\
& \times\left(\left[\left\|f_{1}\left(s, y_{s}+\varphi_{s}, \int_{0}^{s}(s-\gamma)^{(\alpha-1)}(y+\varphi)(\gamma) d \gamma\right)\right\|\right.\right. \\
& \times\left\{\| f_{2}\left(s, y_{\rho(s)}+\varphi_{\rho(s)}, \int_{0}^{s} h\left(\gamma, y_{\gamma}+\varphi_{\gamma}\right) d \gamma\right)\right. \\
& \left.\left.-f_{2}\left(s, z_{\rho(s)}+\varphi_{\rho(s)}, \int_{0}^{s} h\left(\gamma, z_{\gamma}+\varphi_{\gamma}\right) d \gamma\right) \|\right\}\right] \\
& +\left[\left\|f_{2}\left(s, z_{\rho(s)}+\varphi_{\rho(s)}, \int_{0}^{s} h\left(\gamma, z_{\gamma}+\varphi_{\gamma}\right) d \gamma\right)\right\|\right. \\
& \times\left\{\| f_{1}\left(s, y_{s}+\varphi_{s}, \int_{0}^{s}(s-\gamma)^{(\alpha-1)}(y+\varphi)(\gamma) d \gamma\right)\right. \\
& \left.\left.\left.-f_{1}\left(s, z_{s}+\varphi_{s}, \int_{0}^{s}(s-\gamma)^{(\alpha-1)}(z+\varphi)(\gamma) d \gamma\right) \|\right\}\right] d s\right) \\
& \leq\left(g_{1} n_{T}+\frac{T^{\alpha}}{\Gamma(\alpha+1)}\left(f_{11} \mu_{1}+f_{12} \mu_{3}+f_{1}^{*}\right)\left(f_{21} n_{T}+T f_{22} h_{1} n_{T}\right)\right. \\
& \left.+\frac{T^{\alpha}}{\Gamma(\alpha+1)}\left(f_{21} \mu_{2}+f_{22} \mu_{4}+f_{2}^{*}\right)\left(f_{11} n_{T}+\frac{T^{\alpha}}{\alpha} f_{12}\right)\right)\|y-z\|_{\hat{\Omega}},
\end{aligned}
$$


since

$$
\begin{aligned}
\left\|y_{s}-z_{S}\right\|_{B} & \leq m_{T}\left\|y_{0}-z_{0}\right\|_{B}+n_{T} \sup _{0 \leq \eta \leq s}\|y(\eta)-z(\eta)\| \\
& \leq n_{T}\|y-z\|_{\hat{\Omega}},
\end{aligned}
$$

and

$$
\begin{aligned}
\left\|y_{\rho(s)}-z_{\rho(s)}\right\|_{B} & \leq\left(m_{T}+\boldsymbol{J}^{\left(y_{0}-z_{0}\right)}\right)\left\|y_{0}-z_{0}\right\|_{B}+n_{T} \sup _{0 \leq \eta \leq s}\|y(\eta)-z(\eta)\| \\
& \leq n_{T}\|y-z\|_{\hat{\Omega}} .
\end{aligned}
$$

Therefore,

$$
\|\bar{N} y-\bar{N} z\|_{\hat{\Omega}} \leq \lambda_{1}\|y-z\|_{\hat{\Omega}}
$$

which under the assumption $\lambda_{1}<1, \bar{N}$ is acontraction mapping.

Finally, we deduce that $\bar{N}$ has a unique fixed point by means of the Banach fixed point theorem.

Theorem 3.3. Suppose that conditions $\left(H_{1}\right)$ and $\left(H_{2}\right)$ and

$$
\lambda_{2}=n_{T} g_{1}<1
$$

are satisfied, then problem (1.1) has at least one solution.

Proof. For $t \in J$, we decompose $\bar{N}$ as $\bar{N}_{1}+\bar{N}_{2}$, where

$$
\bar{N}_{1} y(t)=-g\left(0, x_{\rho(0)}\right)+g\left(t, y_{\rho(t)}+\varphi_{\rho(t)}\right),
$$

and

$$
\begin{aligned}
\bar{N}_{2} y(t)=\frac{1}{\Gamma(\alpha)} & \int_{0}^{t}(t-s)^{(\alpha-1)}\left(f_{1}\left(s, y_{s}+\varphi_{s}, \int_{0}^{s}(s-\gamma)^{(\alpha-1)}(y+\varphi)(\gamma) d \gamma\right)\right. \\
& \left.\times f_{2}\left(s, y_{\rho(s)}+\varphi_{\rho(s)}, \int_{0}^{s} h\left(\gamma, y_{\gamma}+\varphi_{\gamma}\right) d \gamma\right)\right) d s
\end{aligned}
$$

Next, we are going to show that $\bar{N}_{1}+\bar{N}_{2}$ has a fixed point in $D_{R}$. We classify the proof into the following several steps.

Step 1. Repeating arguments similar to Theorem (3.2), it can easily be proved that

$$
\left(\bar{N}_{1}+\bar{N}_{2}\right)\left(D_{R}\right) \subseteq D_{R}
$$

Step 2. We are going to demonstrate that the map $\bar{N}_{1}$ is a contraction mapping. Letting $y, z \in D_{R}$ and $t \in[0, T]$, we get

$$
\left\|\bar{N}_{1} y(t)-\bar{N}_{1} z(t)\right\| \leq\left\|g\left(t, y_{\rho(t)}+\varphi_{\rho(t)}\right)-g\left(t, z_{\rho(t)}+\varphi_{\rho(t)}\right)\right\| \leq n_{T} g_{1}\|y-z\|_{\hat{\Omega}},
$$

that is,

$$
\left\|\bar{N}_{1} y-\bar{N}_{1} z\right\|_{\hat{\Omega}} \leq \lambda_{2}\|y-z\|_{\hat{\Omega}}
$$

where $\lambda_{2}<1$ is given. 
Step 3. $\bar{N}_{2}$ is a completely continuous operator. The continuity of $f_{1}, f_{2}$ implies that the operator $\bar{N}_{2}$ is continuous. Also we prove that $\bar{N}_{2}$ is uniformly bounded on $D_{R}$.

$$
\begin{aligned}
&\left\|\bar{N}_{2} y(t)\right\| \leq \frac{1}{\Gamma(\alpha)} \int_{0}^{t}(t-s)^{\alpha-1}\left(\left\|f_{1}\left(s, y_{s}+\varphi_{s}, \int_{0}^{s}(s-\gamma)^{(\alpha-1)}(y+\varphi)(\gamma) d \gamma\right)\right\|\right. \\
&\left.\quad \times\left\|f_{2}\left(s, y_{\rho(s)}+\varphi_{\rho(s)}, \int_{0}^{s} h\left(\gamma, y_{\gamma}+\varphi_{\gamma}\right) d \gamma\right)\right\|\right) d s \\
& \leq \frac{T^{\alpha}}{\Gamma(\alpha+1)}\left(f_{11} \mu_{3}+f_{12} \mu_{2}+f_{1}^{*}\right)\left(f_{21} \mu_{1}+f_{22} \mu_{4}+f_{2}^{*}\right)=\eta * .
\end{aligned}
$$

Hence, $\left\|\bar{N}_{2} y\right\|_{\hat{\Omega}} \leq \eta *$, and $\left\{\bar{N}_{2} y(t): y(t) \in D_{R}\right\}$ is uniformly bounded

Now, we prove that $\left\{\bar{N}_{2} y(t): y(t) \in D_{R}\right\}$ is equicontinuous. For every $0 \leq t_{1} \leq t_{2} \leq T$, we obtain

$$
\begin{aligned}
& \left\|\bar{N}_{2} y\left(t_{2}\right)-\bar{N}_{2} y\left(t_{1}\right)\right\| \\
& \leq \frac{1}{\Gamma(\alpha)} \int_{0}^{t_{1}}\left[\left(t_{2}-s\right)^{\alpha-1}-\left(t_{1}-s\right)^{\alpha-1}\right] \\
& \quad \times\left(\left\|f_{1}\left(s, y_{s}+\varphi_{s}, \int_{0}^{s}(s-\gamma)^{(\alpha-1)}(y+\varphi)(\gamma) d \gamma\right)\right\|\right. \\
& \left.\quad \times\left\|f_{2}\left(s, y_{\rho(s)}+\varphi_{\rho(s)}, \int_{0}^{s} h\left(\gamma, y_{\gamma}+\varphi_{\gamma}\right) d \gamma\right)\right\|\right) d s \\
& +\frac{1}{\Gamma(\alpha)} \int_{t_{1}}^{t_{2}}\left(t_{2}-s\right)^{\alpha-1}\left(\left\|f_{1}\left(s, y_{s}+\varphi_{s}, \int_{0}^{s}(s-\gamma)^{(\alpha-1)}(y+\varphi)(\gamma) d \gamma\right)\right\|\right. \\
& \left.\quad \times\left\|f_{2}\left(s, y_{\rho(s)}+\varphi_{\rho(s)}, \int_{0}^{s} h\left(\gamma, y_{\gamma}+\varphi_{\gamma}\right) d \gamma\right)\right\|\right) d s \\
& \leq\left[\int_{0}^{t_{1}}\left[\left(t_{2}-s\right)^{\alpha-1}-\left(t_{1}-s\right)^{\alpha-1}\right] d s+\int_{t_{1}}^{t_{2}}\left(t_{2}-s\right)^{\alpha-1} d s\right] \\
& \quad \times \frac{1}{\Gamma(\alpha)}\left(f_{11} \mu_{3}+f_{12} \mu_{2}+f_{1}^{*}\right)\left(f_{21} \mu_{1}+f_{22} \mu_{4}+f_{2}^{*}\right) \\
& =\frac{1}{\Gamma(\alpha+1)}\left(f_{11} \mu_{3}+f_{12} \mu_{2}+f_{1}^{*}\right)\left(f_{21} \mu_{1}+f_{22} \mu_{4}+f_{2}^{*}\right)\left(t_{2}^{\alpha}-t_{1}^{\alpha}\right) .
\end{aligned}
$$

As $t_{1} \rightarrow t_{2}$, the right-hand side of the above inequality tends to zero. It means that $\left\{\bar{N}_{2} y(t): y(t) \in D_{R}\right\}$ is equicontinuous. Also, the Arzela-Ascoli theorem implies that $\bar{N}_{2}$ is a completely continuous operator. Thus, by the Krasnoselskii's fixed point theorem, we realize that problem (1.1) has at least one solution. This completes the proof.

\section{Applications}

To explain the obtained analytical results, we consider the following functional equation:

$$
\begin{aligned}
{ }^{c} D^{\alpha} & {\left[u(t, x)-\int_{-\infty}^{t} \varphi_{1}(t, s-t, x) Q_{1}\left(u\left(s-\rho_{1}(t), x\right)\right) d s\right] } \\
= & {\left[\int_{-\infty}^{t} \varphi_{2}(t, s-t, x) Q_{2}(u(s, x)) d s+\int_{0}^{t}(t-s)^{\alpha-1} u(s, x) d s\right] } \\
& \times\left[\int_{-\infty}^{t} \varphi_{3}(t, s-t, x) P_{1}\left(u\left(s-\rho_{1}(t), x\right)\right) d s+\int_{0}^{t} \int_{-\infty}^{s} \varphi_{4}(s, \tau-s, x) P_{2}(u(\tau, x)) d \tau d s\right],
\end{aligned}
$$




$$
\begin{aligned}
& u(t, 0)=u(t, \pi)=0, \quad t \in[0, T], \\
& u(t, x)=\phi(t, x), \quad t \in[0, T], \quad x \in[0, \pi],
\end{aligned}
$$

for $0<\alpha \leq 1$ and $\phi \in B$. Let $X=L^{2}[0, \pi]$ with norm $\|\cdot\|_{L^{2}}$. We consider the phase space

$$
\begin{aligned}
& B=\left\{\phi:(-\infty, 0] \rightarrow X, \int_{-\infty}^{0} h(s)\|\phi\|_{[s, 0]} d s<\infty\right\}, \\
& \|\phi\|_{B}=\int_{-\infty}^{0} h(s)\|\phi\|_{[s, 0]} d s,
\end{aligned}
$$

where $\|\phi\|_{[s, 0]}=\sup _{t \in[s, 0]}\|\phi(t)\|$ and $h:(-\infty, 0] \rightarrow(0, \infty)$ is a continuous function with $l=\int_{-\infty}^{0} h(s) d s<$ $\infty$. Choosing $h(s)=e^{2 s}$, for $s<0, l=\frac{1}{2}$.

For $(t, \phi) \in[0, T] \times B$, we consider $\phi(\theta)(x)=\phi(\theta, x),(\theta, x) \in(-\infty, 0] \times[0, \pi]$, and setting $u(t)(x)=$ $u(t, x), \rho(t)=t-\rho_{1}(t)$, thus we have

$$
\begin{aligned}
& g(t, \phi)(x)=\int_{-\infty}^{0} \varphi_{1}(t, \theta, x) Q_{1}(\phi(\theta)(x)) d \theta, \\
& f_{1}(t, \phi, K u(t))(x)=\int_{-\infty}^{0} \varphi_{2}(t, \theta, x) Q_{2}(\phi(\theta)(x)) d \theta+K u(t)(x), \\
& f_{2}(t, \phi, H \phi)(x)=\int_{-\infty}^{0} \varphi_{3}(t, \theta, x) P_{1}(\phi(\theta)(x)) d \theta+H \phi(x),
\end{aligned}
$$

where

$$
\begin{aligned}
& K u(t)(x)=\int_{0}^{t}(t-s)^{(\alpha-1)} u(s)(x) d s \\
& H \phi(x)=\int_{0}^{t} \int_{-\infty}^{0} \varphi_{4}(t, \theta, x) P_{2}(\phi(\theta)(x)) d \theta d s .
\end{aligned}
$$

Along with these configurations, problem (4.1)-(4.2) can be written in the abstract form of the equation (1.1).

Furthermore, suppose that:

(i) The functions $Q_{i}, P_{i}, i=1,2$, are continuous functions and $\phi_{1}(\theta, x), \phi_{2}(\theta, x)$ are the continuous functions in $(-\infty, 0] \times[0, \pi]$, such that

$$
\begin{aligned}
& 0 \leq Q_{i}\left(\phi_{1}(\theta)(x)\right)-Q_{i}\left(\phi_{2}(\theta)(x)\right) \leq \int_{-\infty}^{0} e^{2 s}\left\|\phi_{1}(s, .)-\phi_{2}(s, .)\right\|_{L_{2}} d s, \\
& 0 \leq P_{i}\left(\phi_{1}(\theta)(x)\right)-P_{i}\left(\phi_{2}(\theta)(x)\right) \leq \int_{-\infty}^{0} e^{2 s}\left\|\phi_{1}(s, .)-\phi_{2}(s, .)\right\|_{L_{2}} d s .
\end{aligned}
$$

(ii) The functions $\varphi_{i}(t, \theta, x), i=1,2, \cdots, 4$, are continuous on $[0, T] \times(-\infty, 0] \times[0, \pi]$, and satisfy

$$
\int_{-\infty}^{0} \varphi_{i}(t, \theta, x) d \theta=p_{i}(t, x), \quad\left(\int_{0}^{\pi} p_{i}^{2}(t, x) d x\right)^{\frac{1}{2}}=L_{i}(t),
$$

where $L_{i}(t) \in C[J,(0, \infty)]$ and $l_{i}=\sup _{t \in J}\left|L_{i}(t)\right|<\infty$.

(iii) The function $\rho_{1}:[0, T] \rightarrow[0, \infty)$ is continuous. 
Thus, under the all above conditions, we have

$$
\begin{aligned}
& \left\|g\left(t, \phi_{1}\right)-g\left(t, \phi_{2}\right)(x)\right\|_{L_{2}} \\
& \left.\leq\left[\int_{0}^{\pi}\left\{\int_{-\infty}^{0} \varphi_{1}(t, \theta, x) Q_{1}\left(\phi_{1}(\theta)-\phi_{2}(\theta)\right)(x) d \theta\right)\right\}^{2} d x\right]^{\frac{1}{2}} \\
& \leq\left[\int_{0}^{\pi}\left\{\int_{-\infty}^{0} \varphi_{1}(t, \theta, x) \int_{-\infty}^{0} e^{2 s}\left\|\phi_{1}(s, .)-\phi_{2}(s, .)\right\|_{L_{2}} d s d \theta\right\}^{2} d x\right]^{\frac{1}{2}} \\
& \leq\left[\int_{0}^{\pi}\left\{\int_{-\infty}^{0} \varphi_{1}(t, \theta, x) \int_{-\infty}^{0} e^{2 s} \sup _{\psi \in[s, 0]}\left\|\phi_{1}(\psi)-\phi_{2}(\psi)\right\|_{L_{2}} d s d \theta\right\}^{2} d x\right]^{\frac{1}{2}} \\
& \leq\left[\int_{0}^{\pi}\left\{\int_{-\infty}^{0} \varphi_{1}(t, \theta, x) d \theta\right\}^{2} d x\right]^{\frac{1}{2}}\left\|\phi_{1}-\phi_{2}\right\|_{B}=l_{1}\left\|\phi_{1}-\phi_{2}\right\|_{B} .
\end{aligned}
$$

Therefore, hypothesis $\left(H_{1}\right)$ holds. Similarly, we have

$$
\begin{aligned}
\| f_{1}( & \left.t, \phi_{1}, K u_{1}(t)\right)-f_{1}\left(t, \phi_{2}, K u_{2}(t)\right)(x) \|_{L_{2}} \\
\leq & {\left.\left[\int_{0}^{\pi}\left\{\int_{-\infty}^{0} \varphi_{2}(t, \theta, x) Q_{2}\left(\phi_{1}(\theta)-\phi_{2}(\theta)\right)(x) d \theta\right)\right\}^{2} d x\right]^{\frac{1}{2}} } \\
& +\left[\int_{0}^{\pi}\left\{K u_{1}(t)(x)-K u_{2}(t)(x)\right\}^{2} d x\right]^{\frac{1}{2}} \\
\leq & {\left[\int_{0}^{\pi}\left\{\int_{-\infty}^{0} \varphi_{2}(t, \theta, x) \int_{-\infty}^{0} e^{2 s} \sup _{\psi \in[s, 0]}\left\|\phi_{1}(\psi)-\phi_{2}(\psi)\right\|_{L_{2}} d s d \theta\right\}^{2} d x\right]^{\frac{1}{2}} } \\
& +\left\|K u_{1}(t)-K u_{2}(t)\right\|_{L_{2}} \\
\leq & {\left[\int_{0}^{\pi}\left\{\int_{-\infty}^{0} \varphi_{2}(t, \theta, x) d \theta\right\}^{2} d x\right]^{\frac{1}{2}}\left\|\phi_{1}-\phi_{2}\right\|_{B}+\left\|K u_{1}(t)-K u_{2}(t)\right\|_{L_{2}} } \\
= & l_{2}\left\|\phi_{1}-\phi_{2}\right\|_{B}+l^{*}\left\|K u_{1}(t)-K u_{2}(t)\right\|_{L_{2}},
\end{aligned}
$$

therefore $f_{1}$ satisfies $\left(H_{2}\right)$. Similarly, we can show such results for $f_{2}$. All conditions of the required theorems are now fulfilled. So, we deduce that the problem (4.1)-(4.2) has a unique solution on $[0, T]$.

\section{CONCLUSION}

In this work, we presented some new general sufficient conditions which certify the existence and uniqueness of solutions for the special class of fractional integro-differential equations with time-dependent delay. By means of the Banach's and Krasnoselskii's fixed point theorems, we analyzed the desired model which includes the product of two nonlinear operators. An example is presented to illustrate the importance of the obtained results.

\section{REFERENCES}

[1] R. Hilfer, Applications of Fractional Calculus in Physics, World Scientific, Singapore, 2000. 
[2] K. Nouri, S. Elahi-Mehr, L. Torkzadeh, Investigation of the behavior of the fractional Bagley-Torvik and Basset equations via numerical inverse laplace transform, Rom. Rep. Phys. 68 (2016), 503-514.

[3] Y. Yu, P. Perdikaris, G.E. Karniadakis, Fractional modelling of viscoelasticity in 3D cerebral arteries and aneurysms, J. Comput. Phys. 323 (2016), 219-242.

[4] C. Coussot, Fractional derivative models and their use in the characterization of hydropolymer and invivo breast tissue viscoelasticity, Master Thesis, University of Illiniois at Urbana-Champain, 2008.

[5] A.A. Killbas, H.M. Srivastava, J.J. Trujillo, Theory and Applications of Fractional Differential Equations, Elsevier, Amesterdam, 2006.

[6] I. Podlubny, Fractional Differential Equations, Academic Press, San Diego, 1999.

[7] H. Jafari, C.M. Khalique, M. Nazari, An algorithm for the numerical solution of nonlinear fractional-order Van der Pol oscillator equation, Math. Comput. Modelling 55 (2012), 1782-1786.

[8] H. Jafari, C. M. Khalique, M. Nazari, Application of the Laplace decomposition method for solving linear and nonlinear fractional diffusion-wave equations, Appl. Math. Lett. 24 (2011), 1799-1805.

[9] S. Abbas, M. Benchohra, J.E. Lazreg, J.J. Nieto, On a coupled system of Hilfer and Hilfer-Hadamard fractional differential equations in Banach spaces, J. Nonlinear Funct. Anal. (2018), Article ID 12.

[10] H. Jafari, M. Nazari, D. Baleanu, C.M. Khalique, A new approach for solving a system of fractional partial differential equations, Comput. Math. Appl. 66 (2013), 838-843.

[11] K. Maleknejad, K. Nouri, L. Torkzadeh, Operational matrix of fractional integration based on the shifted second kind Chebyshev polynomials for solving fractional differential equations, Mediterr. J. Math. 13 (2016), 1377-1390.

[12] M. Adimy, F. Crauste, M.L. Hbid, R. Qesmi, Stability and Hopf bifurcation for a cell population model with statedependent delay, SIAM J. Appl. Math. 77 (2009), 1611-1633.

[13] F. Chen, D. Sun, J. Shi, Periodicity in a food-limited population model with toxicants and state dependent delays, J. Math. Anal. Appl. 288 (2003), 136-146.

[14] P. Getto, M. Waurick, A differential equation with state-dependent delay from cell population biology, J. Differential Equations 260 (2016), 6176-6200.

[15] F. Hartung, T. Krisztin, H. Walther, J. Wu, Functional differential equations with state-dependent delays: theory and applications, in: A. Canada, P. Drabek, A. Fonda (Eds.), Handbook of Differential Equations: Ordinary Differential Equations, Elsevier B.V., 2006

[16] K. Nandakumar, M. Wiercigroch, Galerkin projections for state-dependent delay differential equations with applications to drilling, Appl. Math. Model. 37 (2013), 1705-1722.

[17] M. Benchohra, S. Litimein, J.J. Trujillo, M.P. Velasco, Abstract fractional integro-differential equations with statedependent delay, Int. J. Evol. Equ. 6 (2012), 25-38.

[18] F. Hartung, On differentiability of solutions with respect to parameters in neutral differential equations with statedependent delays, Ann. Mat. Pura Appl. 192 (2013), 17-47.

[19] P. Kalamani, D. Baleanu, S. Selvarasu, M. Mallika-Arjunan, On existence results for impulsive fractional neutral stochastic integro-differential equations with nonlocal and state-dependent delay conditions, Adv. Difference Equ. 2016 (2016), Article ID 163.

[20] S. Suganya, M.M. Arjunan, J.J. Trujillo, Existence results for an impulsive fractional integro-differential equation with state-dependent delay, Appl. Math. Comput. 266 (2015), 54-69.

[21] K. Nouri, M. Nazari, B. Keramati, Existence results for a coupled system of fractional integro-differential equations with time-dependent delay, J. Fixed Point Theory Appl. 19 (2017), 2927-2943.

[22] E. Hernandez, A. Prokopczyk, L. Ladeira, A note on partial functional differential equations with state-dependent delay, Nonlinear Anal. Real World Appl. 7 (2006), 510-519.

[23] J. Hale, J. Kato, Phase space for retarded equations with infinite delay, Funkcial. Ekvac. 21 (1978), 11-41. 